Cómo citar este artículo: Ayala de la Rosa, D. M., Figueroa Fonseca, D. M. \& Salcedo Mojica, J. (2017, julio-diciembre). Sistematización de experiencias de algunas universidades privadas de Bogotá sobre su proceso de implementación de Normas Internacionales de Información Financiera. Rev. Cient. Gen. José María Córdova, 15(20), 291-307. DOI: http://dx.doi.org/10.21830/19006586.184

\title{
Sistematización de experiencias de algunas universidades privadas de Bogotá sobre su proceso de implementación de Normas Internacionales de Información Financiera*
}

DOI: http://dx.doi.org/10.21830/19006586.184

Recibido: 26 de octubre de 2016 Aceptado: 5 de junio de 2017

Systematization of Experiences of some Private Universities of Bogota on their Implementation Process of International Financial Reporting Standards

Systématisation d'expériences de certaines universités privées à Bogotá sur le processus de mise en oeuvre des normes internationales d'information financière

Sistematização de experiências de algumas universidades privadas de Bogotá sobre seu processo de implementação de Normas Internacionais de Informação Financeira

Diana María Ayala de la Rosa ${ }^{a}$

Diana Marcela Figueroa Fonseca ${ }^{b}$

Jesús Salcedo Mojica ${ }^{c}$

* El artículo es producto del proyecto de investigación "Análisis de la estructura financiera y administrativa de las Instituciones Privadas de Educación Superior en Bogotá a la luz de las Normas Internacionales de Información Financiera", que se desarrolló entre agosto de 2014 y julio de 2016 por la Facultad de Contaduría Pública y la Facultad de Administración de Empresas de la Universidad Antonio Nariño.

a Universidad Antonio Nariño, Bogotá, Colombia. Master of Science in Accounting de Boston College, Estados Unidos.<dianayala@uan.edu.co>.

b Universidad Antonio Narińo, Bogotá, Colombia. Master of Business Administration, MBA, de la Universidad Viña del Mar, Chile. <dianafigueroa@uan.edu.co>.

c Universidad Antonio Nariño, Bogotá, Colombia. Master of Business Administration, MBA, de la Universidad de Wales, Reino Unido.<jesussalcedo@uan.edu.co>. 
Resumen. Aunque las Instituciones de Educación Superior son entidades sin ánimo de lucro de gran importancia para el desarrollo de los países, se encuentra muy poca evidencia empírica sobre sus normas contables y financieras. En ese sentido, este artículo presenta una sistematización de la experiencia sobre el proceso de implementación de Normas Internacionales de Información Financiera (NIIF) en 17 universidades privadas en Bogotá. Se trata de una investigación de tipo exploratorio organizada de la siguiente manera: en la primera parte se encuentra una revisión general del marco teórico, seguido de los aspectos metodológicos. Los resultados de la investigación se describen en la tercera parte, en donde se puede concluir el profundo efecto que ha tenido la implementación de NIIF en las universidades privadas, pues ha llevado a una reflexión sobre la necesidad de un reporte financiero transparente hacia la sociedad, que prácticamente no existía bajo norma local.

Palabras clave: implementación de NIIF; Instituciones de Educación Superior; Normas Internacionales de Información Financiera.

Abstract. Higher Education Institutions are non-for profit organizations of critical importance for the global development, however there is little empirical evidence about their accounting rules. In that sense, this article presents a systematization of the experience of 17 private universities in Bogotá implementation process of International Financial Reporting Standards - IFRS. This research is exploratory and is organized as follows: In the first section, there is a theoretical framework followed by a description of the methodological aspects. The results are in the third section, where it's important to highlight the profound effect that the IFRS implementation process has had on private universities, because it has cause to reflect on the need for a transparent financial report to the society, that didn't exist under the local regulations.

Keywords: IFRS Implementation; International Financial Reporting Standards; Higher Education Institutions.

Résumé. Les établissements d'enseignement supérieur sont des organismes sans but lucratif qui sont d'une grande importance pour le développement des pays, cependant, nous n'avons pas beaucoup de preuves empiriques sur ses normes comptables et financières. En ce sens, cet article présente une systématisation de l'expérience sur le processus de mise en œuvre des normes internationales d'information financière (IFRS) dans 17 universités privées à Bogotá. Compte tenu de ces actions préparatoires, cette recherche est considérée comme exploratoire. La première partie est une révision générale du cadre théorique, suivi des aspects méthodologiques. Nous trouverons les résultats de cette recherche dans la troisième partie, où l'on peut conclure l'effet de la mise en œuvre des IFRS dans les universités privées et nous réfléchissons à la nécessité d'une information financière transparente pour la société, pratiquement inexistante sous l'application de la norme locale.

Mots-clés: établissements d'enseignement supérieur; Normes internationales d'information financière (NIIF); mise en œuvre des IFRS.

Resumo. Os estabelecimentos de ensino superior são organizaçôes sem fins lucrativos são da maior importância para o desenvolvimento dos países, contudo, não temos muitos elementos de prova empíricos sobre as suas normas contabilísticas e financeiras. Nesse sentido, este artigo apresenta uma sistematização da experiência nos processos de execução das normas internacionais de relato financeiro (IFRS), em 17 Universidades privadas de Bogotá. Tendo em conta essas açôes preparatórias, esta pesquisa é considerada como exploratória. A primeira parte é uma revisão global do quadro teórico, e dos aspectos metodológicos. Nós encontramos os resultados na terceira parte desta pesquisa, onde pode concluir-se o grande impacto da aplicação de NIIF nas Universidades privadas, e refletimos sobre a necessidade de informação financeira transparente para a Sociedade, que praticamente não existe no âmbito de aplicação da regulamentação local.

Palavras chave: aplicação de NIIF; estabelecimentos de ensino superior; Normas internacionais de relato financeiro (NIIF). 


\section{Introducción}

La convergencia a Normas Internacionales de Información Financiera (NIIF) es hoy una realidad en nuestro país que debe asumirse como un proceso complejo que poco a poco va generando cambios en el interior de las organizaciones. Como lo expone Marín Vallespin (2009), la rendición de cuentas (accountability) de las instituciones de educación superior es un fenómeno que se encuentra en la agenda política en materia de educación de muchos países. Por lo tanto, son un interesante objeto de estudio, por ser las instituciones en donde se forma el ciudadano y el profesional, además de que son un eje fundamental en la actual sociedad de la globalización y el conocimiento.

El objetivo fundamental de este trabajo es conocer las principales características del proceso de implementación de las NIIF en las universidades privadas. Se considera exploratorio, dado que existe muy poca literatura al respecto, en cuanto las universidades son generalmente más estudiadas desde su aspecto académico, obviando su importancia como organizaciones que administran importantes recursos para el servicio público de la educación. Se utilizó como instrumento de recolección de información la entrevista semiestructurada, la cual permite observar los fenómenos con fundamento en el enfoque de la contabilidad social, desde la cual la realidad económica se construye socialmente y por lo tanto es relevante conocer los puntos de vista de los agentes que intervienen en la elaboración de la información financiera y realizar los análisis a partir de sus respuestas.

Dado lo anterior, este artículo se encuentra dividido en cuatro partes. En la primera se hace una exposición del marco teórico, compuesto por tres ejes esenciales: la normatividad sobre NIIF en nuestro país, la literatura al respecto del proceso de implementación y una descripción del sistema de Educación Superior en Colombia. En la segunda parte se describen los aspectos metodológicos de la investigación. En la tercera se encuentra el análisis de las respuestas obtenidas en las entrevistas, en tanto que en la parte final se hacen recomendaciones y conclusiones que han surgido de este trabajo.

\section{Marco teórico y antecedentes}

\section{Implementación de Normas Internacionales de Información Financiera}

La comunidad internacional, a través del International Accounting Standards Board (IASB), inició un proceso complejo y global al desarrollar parámetros o estándares internacionales de información financiera que permitieran lograr una articulación entre todos los países en búsqueda de un lenguaje contable común, de tal forma que con el tiempo hicieran posible derogar las normativas nacionales y allanar el camino para una armonización mundial.

Más de cien países han acogido la iniciativa de adopción o convergencia a las Normas Internacionales, y ello constituye una de sus potencialidades, ya que su aceptación mundial posibilita el acceso a los mercados internacionales, lo cual es fundamental en la era de la globalización y la internacionalización. 
Con la expedición de la Ley 1314 de 2009, el Estado colombiano tomó el camino de la convergencia a normas de contabilidad, de información financiera y de aseguramiento de la información. En diciembre de 2015, el Ministerio de Comercio, Industria y Turismo expidió los decretos 2420 y 2496 con el fin de compilar las normas reglamentarias preexistentes sobre información financiera y de aseguramiento de la información.

Para la convergencia a NIIF, se determinaron tres grupos con características clave que deben ser analizadas por las empresas para saber cuál normativa tienen que acoger. El grupo uno debe cumplir con las NIIF plenas, el grupo dos atenderá a las NIIF para pequeñas y medianas entidades (NIIF para pymes), y el grupo tres, por su parte, deberá implementar un modelo de contabilidad simplificada basada en el sistema de causación, atendiendo a las pautas del Intergovernmental Working Group of Experts on International Standards of Accounting and Reporting (ISAR) (Ferrer de la Hoz, 2013, p. 982).

El cronograma establecido por el Gobierno para la aplicación de las NIIF contempla que en el año 2016 todas las organizaciones debían estar aplicando los estándares internacionales para el registro y reporte de su información financiera.

\section{Normatividad sobre las Instituciones de Educación Superior en Colombia}

De acuerdo con la Ley 30 de 1992, por la cual se organiza el servicio público de la Educación Superior en Colombia, las Instituciones de Educación Superior (IES) son entidades que cuentan con el reconocimiento oficial como prestadoras del servicio público de la educación y se encuentran clasificadas de la siguiente manera:

a. Instituciones Técnicas Profesionales: aquellas facultadas legalmente para ofrecer programas de formación en ocupaciones de carácter operativo e instrumental y de especialización en su respectivo campo de acción, sin perjuicio de los aspectos humanísticos propios de este nivel.

b. Instituciones Universitarias o Escuelas Tecnológicas: aquellas facultadas para adelantar programas de formación en ocupaciones, programas de formación académica en profesiones o disciplinas y programas de especialización.

c. Universidades: las reconocidas actualmente como tales y las instituciones que acrediten su desempeño con criterio de universalidad en las siguientes actividades: la investigación científica o tecnológica; la formación académica en profesiones o disciplinas y la producción, desarrollo y transmisión del conocimiento y de la cultura universal y nacional.

El Artículo 98 de la Ley 30 de 1992 estipula que las Instituciones privadas de Educación Superior deben constituirse como personas jurídicas de utilidad común, sin ánimo de lucro, organizadas como corporaciones, fundaciones o instituciones de economía solidaria.

Asimismo, el Estatuto Tributario establece que las IES no son contribuyentes del impuesto a la Renta, ni responsables del Impuesto al Valor Agregado (IVA), ni sujetas al Impuesto al Patrimonio. Por lo tanto, no se les deben practicar retenciones en la fuente, y, en consecuencia, 
presentan la declaración de ingresos y patrimonio. Todos estos beneficios tributarios están fundamentados en la prestación del servicio público de la educación, que es una función social del Estado, consagrada en la Constitución Nacional. La información solicitada a estas entidades por parte de la dirección de impuestos es realmente informativa, y esto afecta los incentivos para su fiscalización.

El Consejo Técnico de la Contaduría Pública (CTCP), en una consulta realizada el 15 de agosto de 2008, indicó que en virtud de la Ley 30 de 1992, reglamentada por el Decreto 1478 de 1994, aplicable a las Instituciones privadas de Educación Superior, se determina lo siguiente:

La obligatoriedad de la designación del revisor fiscal, los estatutos se deben ajustar a la legislación mercantil en lo relativo al cumplimiento de funciones, inhabilidades e incompatibilidades, obligaciones e informes, incluso por la remisión a los requisitos de las sociedades anónimas, estas revisorías cuentan para el límite de las cinco establecidas por el artículo 215 del Código de Comercio. (CTCP, 2008, s. p.)

Asimismo, expresó que el Plan Único de Cuentas para Comerciantes es aplicable a todas las personas naturales o jurídicas que estén obligadas a llevar contabilidad para quienes no se hayan expedido planes de cuentas en virtud de legislación especial.

En el concepto del 27 de agosto de 2014, el CTCP especifica que, de acuerdo con las normas colombianas, las entidades que están reguladas por el Ministerio de Educación deben acogerse a la Ley 1314 de 2009, la cual reglamenta a las diferentes entidades en la implementación de las Normas Internacionales de Información Financiera y clasificarse en alguno de los tres grupos definidos en el Direccionamiento del CTCP, considerando los requisitos contemplados en los decretos reglamentarios de la Ley 1314 de 2009.

El Documento de orientación técnica N. 14. Aplicación NIIF en entidades sin ánimo de lucro, brinda un esbozo general sobre los centros educativos y describe que derivan sus recursos de los cobros por matrículas, pensiones, otros servicios y donaciones. Además, describe que suelen tener un nivel significativo de inversiones en inmuebles y en menor nivel en otros activos fijos.

Como lo señalan Giraldo, Abad y Díaz (2007), aunque durante varios años se realizaron muchos proyectos de reformas educativas en el país, es con la "promulgación de la Constitución Política de Colombia en el año de 1991 en la que aparece en el ámbito de la educación el espíritu de la calidad como objetivo rector de los procesos educativos" (p. 1). En 1992, con la Ley 30 se crea el Consejo Nacional de Acreditación (CNA), compuesto por siete académicos para revisar, organizar, fiscalizar, dar fe de la calidad y finalmente recomendar al Ministerio de Educación Nacional la acreditación de los programas e instituciones que lo merezcan.

De esta manera, en los últimos años, algunas entidades universitarias, acudiendo a su autonomía, han tomado la determinación de someterse a los parámetros del CNA para autoevaluarse, mejorar y obtener la acreditación. Una acreditación que "debe ser reconocida y analizada como el ejemplo de lo que debe ser logrado para todas las demás en el largo plazo" (Giraldo, Abad \& Díaz, 2007, p. 12). De acuerdo con el Artículo 1 del Decreto 2904 de 1994, por el cual se reglamenta el Sistema Nacional de Acreditación (Art. 53 y 54 de la Ley 30 de 1992), la acreditación es

el acto por el cual el Estado adopta y hace público el reconocimiento que los pares académicos hacen de la comprobación que efectúa una institución sobre la calidad de sus programas académicos, su organización y funcionamiento y el cumplimiento de su función social. 
Según el Sistema Nacional de Información de la Educación Superior (SNIES) ${ }^{1}$, en la ciudad de Bogotá hay 96 Instituciones privadas de Educación Superior, de las cuales, de acuerdo con el carácter académico, 15 son instituciones técnicas profesionales, 17 instituciones tecnológicas, 40 instituciones universitarias y 24 universidades. Del total de instituciones en Bogotá, solo $11^{2}$ son acreditadas de alta calidad y todas ellas son universidades o instituciones universitarias.

En lo referente a investigación académica sobre las Instituciones de Educación Superior se encuentra que en el ámbito colombiano existen descripciones muy amplias, como en Roa y Pacheco (2014) y Misas (2004), en las que hay un aporte importante para comprender el desarrollo de las instituciones y sus principales características. Pinto, Becerra y Gómez (2013) realizan una descripción del financiamiento de la educación superior y Católico (2012) presenta una revisión sobre el uso de las herramientas de gerencia estratégica de costos en las IES privadas. Sin embargo, se debe advertir que no se encuentra literatura académica que analice en detalle la información financiera de las IES privadas, ni tampoco sobre la implementación de las NIIF en las mismas organizaciones.

\section{Principales impactos de la implementación de las NIIF en Colombia}

La investigación sobre la implementación de las NIIF en nuestro país es abundante. En Salazar (2011) se analizan varias implicaciones de las NIIF para las pymes, dentro de lo cual se resalta el incremento de las conciliaciones contables y tributarias, así como un mayor uso de las mediciones financieras (valor razonable y valor presente). Por su parte, Arias-Bello y Sánchez-Serna (2011) denotan que dentro de los cambios estructurales que traen las NIIF para el país se encuentran la cultura de la información organizacional, el uso de tecnologías de la información, la comprensión de las diferentes partes interesadas, entre otras.

Castaño-Ríos, Correa-García y Zamarra-Londoño (2014) realizan un estudio de caso de una cooperativa en Bogotá y encuentran que para poder responder a los requerimientos de la implementación de las NIIF es necesario incrementar el personal, especialmente del área financiera y contable, así como aumentar los tiempos de capacitación, tanto en el conocimiento de las NIIF, como para el uso del software. Asimismo, observan que durante un tiempo, el software tendrá que soportar la existencia de tres bases diferentes de reporte de información, así: actuales Principios de Contabilidad Generalmente Aceptados (PCGA), NIIF y contabilidad para efectos tributarios. También se destaca que, como primera etapa del proceso, se debe realizar una sensibilización a todo el personal clave de la entidad para que se comprenda que toda la organización queda involucrada en el proceso.

1 El Sistema Nacional de Información de la Educación Superior (SNIES) del Ministerio de Educación Nacional es el conjunto de fuentes, procesos, herramientas y usuarios que, articulados entre sí, posibilitan y facilitan la recopilación, divulgación y organización de la información sobre educación superior relevante para la planeación, monitoreo, evaluación, asesoría, inspección y vigilancia del sector (Art. 1 del Decreto 1767 de 2006).

2 Para la selección de las entidades universitarias se realizó la consulta al SNIES el 30 de septiembre de 2015, en la cual se encontraban acreditadas 11 entidades. Actualmente, hay 12, ya que el 30 de junio de 2016 obtuvo resolución de acreditación la Universidad del Bosque. 
En Díaz (2014) se encuentra de forma concisa una lista de las experiencias vividas en los procesos de convergencia a NIIF, que son muy precisas y sirven como base para la investigación contable sobre los principales puntos a tener en cuenta en la revisión de los impactos reales, así:

- Redefinición de políticas contables a cargo de la alta dirección.

- Definición de plan estratégico para la implementación de las NIIF.

- Evaluación de plataformas tecnológicas para el soporte de reportes acorde con las necesidades.

- Designación de líderes en el manejo del proyecto NIIF.

- Seguimientos a los reportes y compromisos.

- Capacitación y entrenamiento a todo nivel.

- Apoyo en asesoría y consultoría de diferentes disciplinas.

- Establecimiento de los códigos de cuentas a las necesidades de la organización.

- Análisis de los elementos de los estados financieros y evaluación de los impactos al implementar las NIIF.

- Elaboración del Estado Financiero de Apertura.

Como lo señala Ramírez (2015), uno de los efectos más importantes para las empresas en Colombia serán los costos en los que tendrán que incurrir para soportar los cambios asociados al proceso de convergencia. Estos costos se pueden asociar a los honorarios de auditoría, consultorías y reingeniería en procesos y las tecnologías de la información. Los responsables de la convergencia y los directivos de las compañías deben tener en cuenta esto en el momento de estimar los recursos para el proceso.

Mantilla (2014) denota la importancia tanto de los aspectos técnicos de las NIIF como de la utilización de las herramientas de administración de proyectos para lograr la transición desde las normas contables locales hacia la normativa internacional. Asimismo, indica la relevancia de hacer la implementación pasando a través de cuatro "disciplinas": Preparación, Medición, Presentación y Revelación de la información en los estados financieros, que tienen una lógica especial de manera que sea útil para la toma de decisiones.

Cabe resaltar que el paradigma actual de la contabilidad, tanto a nivel local como internacional, está enfocado principalmente a la medición de las ganancias para el inversionista, por lo cual las entidades que no persiguen lucro, como las universidades, han tenido que adaptar su sistema de información financiera con esta limitación. Tal como lo señala el Consejo Técnico de la Contaduría Pública en el Documento de orientación técnica N. ${ }^{\circ} 14$. Entidades Sin Ánimo de Lucro,

las particularidades de las ESAL han llevado a solicitar al IASB la emisión de un estándar internacional de información financiera para ellas, asunto que aún no está en la agenda del emisor internacional, dado que su trabajo se centra en las entidades con ánimo de lucro y especialmente las que participan en mercados de capitales. Un proyecto de esta naturaleza obligaría al IASB a cambiar su constitución, dado que sus actividades se orientan a los mercados de capitales y a las entidades con ánimo de lucro. (CTCP, 2015b) 


\section{Diseño y metodología de la investigación}

La metodología que se utilizó en esta investigación fue cualitativa, pues implicó un proceso de análisis y vinculación de datos recolectados a través de una entrevista semiestructurada, dirigida a los funcionarios encargados de implementar las NIIF en las entidades universitarias. Hay que resaltar que las entrevistas son una herramienta que sirve para "recoger información y comprender los fenómenos desde el punto de vista del sujeto” (Hernández, 2014, p. 188).

Para la presente investigación se seleccionaron veinte instituciones universitarias de la ciudad de Bogotá, teniendo como parámetro primario la acreditación de alta calidad ante el CNA, al considerar que esta característica representa un interés en mejorar sus sistemas de información, dentro de los cuales se encuentra el financiero y contable. De estas instituciones, $17^{3}$ aceptaron participar en el presente estudio y para ello designaron a una persona conocedora del proceso de implementación, que en su mayoría fueron los directores del área contable.

Las entrevistas fueron diseñadas teniendo en cuenta las descripciones de las experiencias vividas en el proceso de implementación descritas en Díaz (2014). También se tuvo en cuenta el Documento de orientación técnica $N .^{\circ}$ 5. Adopción por primera vez de las NIIF para las pymes, del Consejo Técnico de la Contaduría Pública, en el cual se clasifican los efectos potenciales de la adaptación de una entidad a NIIF para las pymes de la siguiente manera:

- Directos: Referentes a los efectos sobre la contabilidad.

- Primarios: Efectos sobre procesos y sistemas.

- Derivados: Efectos sobre la estructura organizativa, los recursos humanos y aspectos de negocio.

\section{Análisis de los resultados}

Cañibano-Calvo (2004), citado por Cuevas-Mejía y Usme-Suárez (2013, p. 1053), indica que la contabilidad no está confinada al reconocimiento de las transacciones y que por el contrario tiene un ámbito mayor al considerarse un bien público, el cual no solo interesa a los administradores y propietarios del capital, sino que debe tener presente la multiplicidad de usuarios interesados en la información que emana de esta. Así las cosas, la preparación de la información de las entidades universitarias está mediada por grupos de interés muy diversos que van desde directivos, estudiantes, docentes y egresados, hasta quienes están interesados en los programas ofrecidos, padres de familia, y la comunidad que convive alrededor de ella. Asimismo, el Estado está interesado en conocer la información, con una mirada diferente con la que vigila a las organizaciones con ánimo de lucro, ya que, en este caso, lo más importante es la política pública en educación.

3 Las Instituciones universitarias que participaron en este estudio fueron: Universidad Javeriana, Universidad de los Andes, Fundación Universitaria de Ciencias de la Salud, Universidad EAN, Escuela Colombiana de Ingeniería Julio Garavito, Universidad Santo Tomas, Universidad Antonio Narińo. Universidad Jorge Tadeo Lozano, Universidad Manuela Beltrán, Universidad Sergio Arboleda, Universidad del Rosario, Universidad ECCI, Universidad Libre, Universidad Gran Colombia, Universidad de la Sabana, Universidad del Bosque y Corporación Universitaria Republicana. 
Asimismo, la contabilidad es el instrumento que apoya la labor de la administración en la planeación, organización y dirección de las empresas. Los procesos de implementación de la normativa internacional suelen considerarse un proyecto de carácter estratégico que involucra a toda la organización y requiere de significativos elementos de liderazgo y cultura empresarial e implican enormes desafíos en cuanto al sentido de pertenencia.

\section{Importancia y relevancia de las NIIF en Colombia y en las universidades}

Se indagó sobre las consideraciones existentes en cuanto a la importancia y la relevancia de la aplicación de NIIF en Colombia y en las universidades. Se observó un consenso entre los entrevistados en torno a considerar que la normativa internacional permite la unificación de criterios contables y por lo tanto se obtiene una mejora en la comparabilidad y la organización de las finanzas.

Se encontraron tres aspectos importantes al respecto:

- La implementación de las NIIF permite hacer una identificación de la situación financiera y contable real de la entidad, teniendo en cuenta que la información se estaba preparando desde una perspectiva fiscal y no financiera, lo cual desviaba la atención a temas meramente tributarios y de interés para la administración de impuestos únicamente, sin tener en cuenta otros usuarios de la información financiera.

- La internacionalización es un asunto que está marcando la tendencia de las instituciones universitarias, ya que se desea participar a nivel global de diversas maneras en cada una de las funciones sustantivas. Se espera que una buena gestión de la información financiera permita a las instituciones mejorar sus posibilidades de integrarse con pares.

- El carácter de entidad sin ánimo de lucro cuyo fin es la educación de los ciudadanos refuerza la idea de una institución con una alta responsabilidad social, que debe responder a este reto con información de alta calidad.

\section{Inicio del proceso de implementación y grupo seleccionado para el reporte}

Todas las instituciones educativas entrevistadas habían iniciado el proceso de implementación de las NIIF, cumpliendo efectivamente con los plazos dados en la normatividad. La mayoría lo inició en el año 2014, para lograr realizar el Estado de Situación Financiera de Apertura (ESFA) a finales del 2015 y comenzar la presentación de Estados Financieros bajo NIIF a partir del 2016.

Por su parte, quince ( $88 \%$ de las instituciones universitarias entrevistadas) se clasificaron en el grupo dos, en razón a que cumplían con las características determinadas por el Consejo Técnico de la Contaduría Pública en el Direccionamiento Estratégico. Solamente dos de dichas instituciones se clasificaron en el grupo uno, una de ellas porque cuenta con una sucursal en el exterior que reporta bajo NIIF, y la otra por decisión de sus directivos. 


\section{Principales procesos afectados con la implementación de las NIIF}

Para identificar los principales procesos que se vieron afectados con la implementación de las NIIF se incluyeron las preguntas 12 y 13, en las cuales se solicitó realizar una breve descripción de las áreas y procesos que tuvieron más cambios con la aplicación de la normatividad internacional. Dicha pregunta está fundamentada en "que la cuenta es el punto inicial de donde parte el proceso de registro de las transacciones" (Consejo Técnico de la Contaduría Pública, 2015b, p. 94).

Las respuestas obtenidas al respecto coinciden en que el principal impacto para las instituciones universitarias se da en los procesos relacionados con la propiedad, planta y equipo, ya que las NIIF exigen un mayor análisis al momento de su reconocimiento, medición y valoración. También señalaron que ya no se trata solamente de un contador que desde su oficina determina unas vidas útiles basándose en la normatividad tributaria, sino que las áreas en el interior de las entidades encargadas de los activos fijos deben participar de forma dinámica en la determinación y cumplimiento de las políticas contables y en la revelación que sobre estos haya que hacerse en los estados financieros.

Asimismo, algunos funcionarios indicaron que con la normatividad internacional hay importantes cambios sobre los procesos relacionados con recursos humanos. Esto debido a que la normatividad internacional exige el análisis, contabilización y revelación de los beneficios que se les dan a los empleados. Es usual que en las instituciones universitarias existan incentivos para los docentes y/o administrativos, que incluyen derecho a descanso remunerado al cumplir cierto tiempo de trabajo en la institución, tales como en el caso de los quinquenios o el año sabático.

Se identificaron también cambios en el reconocimiento de ingresos dadas las particularidades de las entidades universitarias, las cuales reciben las matrículas de manera anticipada.

\section{Implicaciones sobre la estructura organizacional}

Con el objetivo de identificar los principales cambios que ha implicado la implementación de las NIIF en las instituciones educativas, se realizaron las preguntas 6 y 7 , la primera enfocada al área financiera y la segunda sobre la estructura administrativa.

Un total de catorce universidades $(82 \%)$ indicaron que sí hubo un gran impacto en sus áreas financieras, mientras que las restantes tres $(17,6 \%)$ respondieron que no experimentaron grandes cambios. Entre las razones citadas con respecto a los ajustes que debieron realizarse para adaptar el área financiera al proceso de implementación de las NIIF se encuentran las siguientes:

- Se requiere mayor trabajo en equipo, ya que anteriormente el área financiera realizaba su trabajo de manera independiente con respecto a las áreas operativas y con la normatividad internacional se requiere que todas las áreas estén atentas a las políticas contables establecidas y participen activamente para lograr que la información sea reflejada en los estados financieros ajustada a la realidad.

- El enfoque del personal de las diferentes áreas debe ser más analítico, pues ya no se trata de repetir funciones periódicamente, sino que se debe aportar de manera integral desde la perspectiva de cada profesional. 
Con respecto a los cambios en el área administrativa, se encontró que la mitad de los entrevistados opinan que la implementación de las NIIF exige varios cambios, entre los cuales se encuentran:

- El reconocimiento, medición y valoración de los activos fijos tiene un gran impacto en el área administrativa, pues los encargados de estas deben estudiar mejor todo lo relacionado con su representación en los estados financieros. Por esa razón, dichos funcionarios debieron capacitarse a la par con los del área financiera y estar más conscientes de la importancia que tienen para la organización, ya que se trata de uno de los rubros más importantes de los estados financieros de una institución educativa.

- Se requieren nuevos reportes, especialmente como soporte a las revelaciones, lo cual genera una mayor visibilidad de algunos departamentos que permanecían desconectados del área financiera y que realizaban su labor de manera operativa, sin tener en cuenta el impacto de sus operaciones en los estados financieros.

- Con el fin de dar cumplimiento a las políticas contables que ahora son dirigidas y establecidas por los directivos de la entidad y cumplidas por cada área, los controles internos se deben refinar para lograr obtener una imagen fiel de la situación financiera de la institución.

\section{Equipo de trabajo para la implementación de las NIIF}

Con el objetivo de conocer la forma en la cual se estableció el equipo de trabajo para realizar la implementación de las NIIF dentro de las instituciones universitarias, se hicieron las preguntas 9, 15 y 16.

En la entrevista se indagó sobre qué funcionario debería ser quien liderara el proceso de implementación a NIIF, para lo cual se dieron cuatro opciones, entre ellas: el Contador, el Director Financiero, el Director Administrativo, el Rector o Todos los anteriores. La mayoría de las respuestas apuntó a que todos estos funcionarios deberían participar en el liderazgo de dicho proceso.

Para conocer el perfil de quienes participaron en el equipo de trabajo se realizó la pregunta sobre cuáles fueron los profesionales que más intervinieron en el proceso de implementación, dando tres opciones, así: Contadores, Administradores, Abogados, Otros. La mayoría (78\%) coinciden con que todos los profesionales deben participar, entre otras razones debido al requerimiento que establece la norma internacional para que las políticas contables sean definidas desde la dirección y aplicadas por cada uno de los departamentos responsables. De esta manera se recomienda conformar un equipo de trabajo multidisciplinario que lidere el proceso. Asimismo, el $22 \%$ considera que es el contador, con todo su conocimiento y experticia, quien debe estar en la cabeza del proceso, con el respaldo de los directivos. En este caso, haciendo la salvedad de que también es necesario el conocimiento de la institución y de todos sus procesos para ejercer en realidad un liderazgo y no solo conocer la norma contable, pues requiere de mucha especificidad en la aplicación.

También se indagó sobre la conformación del comité de trabajo para la implementación de las NIIF en el interior de las instituciones. En este aspecto se observa básicamente dos caracterís- 
ticas. Por una parte están las instituciones cuyo equipo está conformado fundamentalmente por el área financiera y contable, sujetos a la aprobación de los directivos, y por otra parte están las instituciones que incluyeron además a los responsables de todas las áreas involucradas, tales como tesorería, activos fijos, bibliotecas, compras e informática. Se destaca que en todas las instituciones contaron con la colaboración y asesoría de un consultor externo y de la revisoría fiscal.

\section{Impacto en la tecnología}

Con el objetivo de conocer con detalle el impacto que ha tenido la implementación con respecto a la tecnología, se realizó de manera abierta la pregunta 10. Se destacan las siguientes respuestas:

- El volumen de transacciones realizadas por estas instituciones ameritan un sistema robusto.

- Debe incluir funcionalidades para ayudar a mejorar la forma en la que se realizan las revelaciones y la parametrización de las políticas contables.

- El software debe soportar el manejo de múltiples contabilidades para que la institución pueda manejar la información con visión financiera aparte de la información con visión tributaria. El caso de los activos fijos es uno de los más complejos, ya que la tecnología debe permitir manejos de diferentes vidas útiles de acuerdo con el tipo de contabilidad tributaria o financiera.

\section{Descripción de la experiencia del proceso de implementación de NIIF}

Para conocer una descripción completa del proceso de implementación de la NIIF en las instituciones universitarias, se realizaron las preguntas 14 y 19. Las principales apreciaciones se encaminan en lo siguiente:

- Capacitación: hay que destacar que dado que varias de las universidades cuentan con el programa de Contaduría Pública, gran parte de la capacitación se realizó a través de diplomados desarrollados y diseńados por las mismas instituciones, con lo cual se observa esta importante interrelación entre su objeto de negocio y la implementación de las normas internacionales. También es importante destacar que el proceso requiere preparación, tanto para los administrativos como en algunos casos para la parte académica.

- Trabajo en equipo: se debe trabajar como un proyecto interdisciplinario que involucra muchas áreas.

- Participación de la Dirección en el proceso contable.

- Consultores expertos: el proceso se hace menos complejo y realizable cuando se cuenta con consultores expertos que conocen las metodologías y establecen los tiempos de trabajo.

- Personal con conocimiento de fondo de la institución: el conocimiento de las especificidades de la universidad es un factor de éxito en la implementación. 
- Instituciones con sedes: el proceso de implementación de las NIIF es un poco más complejo al tener que trabajar en equipo y realizar mucha capacitación con respecto al personal que labora en las sedes.

- La creación de una nueva cultura en la que la información financiera es parte de toda la institución y todos deben propender por que su área sea reflejada de manera fiel en los estados financieros y que esto no dependa solamente del contador y de su propia visión, sino de una política de la institución, apoyada desde todas las áreas.

Con respecto a los inconvenientes que se tuvieron para realizar la implementación, once instituciones universitarias (61\%) señalaron que se trataron de tipo técnico, principalmente por el software; tres expresaron que fue de tipo presupuestal (18\%), por cuanto se trata de un cambio bastante fuerte y por lo tanto requirió una gran inversión de parte de las instituciones; y tres $(18 \%)$ aseguraron que tuvieron ambos inconvenientes.

\section{Recomendaciones para quienes comienzan el proceso}

Con el objetivo de obtener una visión de parte de estas instituciones, que ya han iniciado su proceso de implementación, se realizó la pregunta 20. Dentro de las recomendaciones más sobresalientes se encuentran las siguientes:

- Capacitación: proceso clave para lograr una implementación exitosa.

- Responsabilizar a todas las áreas generadoras de información contable.

- Asesores externos con amplia experiencia para guiar el proceso y que sean muy rigurosos en el cumplimiento del calendario.

- Equipo interdisciplinario: conformar un grupo de personas altamente calificadas en NIIF dentro de la institución.

- Involucramiento de la alta dirección.

\section{Caracterización de las experiencias}

Siguiendo el Documento de orientación técnica $N{ }^{\circ} 5$. Adopción por primera vez de las NIIF para las pymes, del Consejo Técnico de la Contaduría Pública, los resultados del presente trabajo se pueden observar en la tabla 1 . 
Tabla 1. Sistematización de experiencias del proceso de implementación de NIIF en universidades privadas

\begin{tabular}{|c|c|c|c|}
\hline $\begin{array}{l}\text { Tipo de } \\
\text { efecto }\end{array}$ & Descripción & Categoría & Observaciones dadas por los entrevistados \\
\hline Directos & $\begin{array}{l}\text { Referentes } \\
\text { a los efectos } \\
\text { sobre la conta- } \\
\text { bilidad }\end{array}$ & $\begin{array}{l}\text { Beneficios } \\
\text { a empleados } \\
\text { a largo plazo }\end{array}$ & $\begin{array}{l}\text { - Hay un mejoramiento en la preparación de la información } \\
\text { financiera con fines de reporte. Las NIIF tienen mayores } \\
\text { exigencias en el reconocimiento, medición y presentación } \\
\text { de las transacciones económicas y contables. } \\
\text { - Vida útil: basada en condiciones reales avaladas por estu- } \\
\text { dios técnicos. } \\
\text { - Revisión anual: del valor del activo, así como de su vida } \\
\text { útil, adiciones y mejoras. } \\
\text { - Revelaciones sobre asuntos significativos. } \\
\text { - Semovientes: revisión para determinar su valoración ini- } \\
\text { cial, vida útil y medición posterior, ya que son utilizados } \\
\text { para propósitos educativos. } \\
\text { - Material bibliográfico: establecer vida útil mediante po- } \\
\text { lítica. } \\
\text { - Reconocimiento, medición y revisión continua. } \\
\text { - Generalmente reconocidos como quinquenios, primas } \\
\text { por antigüedad o ańos sabáticos, dependiendo de la ins- } \\
\text { titución. } \\
\text { - Se debe revisar la esencia de los contratos de arrenda- } \\
\text { miento de bienes inmuebles, para determinar si se trata } \\
\text { de arrendamiento financiero u operativo. } \\
\text { - Establecer las políticas de clasificación, control y segui- } \\
\text { miento sobre los bienes y suministros consumibles uti- } \\
\text { lizados dentro de la universidad, bien sea para prácticas } \\
\text { académicas (tales como reactivos, medicamentos, alimen- } \\
\text { tos para animales y herramientas menores entre otras) o } \\
\text { para su mantenimiento. } \\
\text { la naturaleza del negocio y no como venía establecido tri- } \\
\text { butariamente. }\end{array}$ \\
\hline Primarios & $\begin{array}{l}\text { Efectos sobre } \\
\text { procesos } \\
\text { y sistemas }\end{array}$ & $\begin{array}{l}\text { Tecnología } \\
\text { de la información }\end{array}$ & $\begin{array}{l}\text { - Se debe confeccionar un nuevo catálogo de cuentas que esté } \\
\text { acorde con las NIIF y utilizando como guía el Plan Único } \\
\text { de Cuentas para Instituciones de Educación Superior, emi- } \\
\text { tido por la Contaduría General de la Nación. } \\
\text { - Los cambios de la normativa internacional afectan la } \\
\text { información financiera y por lo tanto su soporte en las } \\
\text { Tecnologías de la Información. } \\
\text { - Se realizó una gran inversión en el área de tecnología para } \\
\text { la implementación de las NIIF. }\end{array}$ \\
\hline
\end{tabular}




\begin{tabular}{|c|c|c|c|}
\hline $\begin{array}{l}\text { Tipo de } \\
\text { efecto }\end{array}$ & Descripción & Categoría & Observaciones dadas por los entrevistados \\
\hline \multirow{5}{*}{ Derivados } & \multirow{5}{*}{$\begin{array}{l}\text { Efectos sobre } \\
\text { la estructura } \\
\text { organizativa, } \\
\text { los recursos } \\
\text { humanos y } \\
\text { aspectos de } \\
\text { negocio }\end{array}$} & De estructura & $\begin{array}{l}\text { - Varias áreas dentro de las universidades generalmente } \\
\text { trabajaban de manera independiente al área contable y fi- } \\
\text { nanciera. Ahora deben participar activamente para el cum- } \\
\text { plimiento de las políticas contables de forma integral. } \\
\text { - Para la implementación se estableció generalmente un } \\
\text { equipo interdisciplinario y en el futuro esto ayudará a to- } \\
\text { mar mejores decisiones. }\end{array}$ \\
\hline & & & $\begin{array}{l}\text { - Capacitación: para la generación, procesamiento y aná- } \\
\text { lisis de la información de acuerdo con la normativa in- } \\
\text { ternacional. }\end{array}$ \\
\hline & & $\begin{array}{l}\text { De recursos } \\
\text { humanos }\end{array}$ & $\begin{array}{l}\text { - Capacidad de trabajo en equipo: tanto el área financiera } \\
\text { como las demás áreas relacionadas con el reporte finan- } \\
\text { ciero, deben establecer procedimientos de trabajo para } \\
\text { lograr mayor colaboración entre áreas e intercambio de } \\
\text { información. }\end{array}$ \\
\hline & & & $\begin{array}{l}\text { - Área jurídica: mayor involucramiento con el área conta- } \\
\text { ble y financiera, especialmente en lo relacionado con liti- } \\
\text { gios para su cuantificación y probabilidad de ocurrencia a } \\
\text { fin de determinar la posible provisión. }\end{array}$ \\
\hline & & $\begin{array}{l}\text { Participación de } \\
\text { las directivas en el } \\
\text { establecimiento de } \\
\text { políticas contables }\end{array}$ & $\begin{array}{l}\text { - La institución debe contar con políticas contables claras, } \\
\text { establecidas en un documento aprobado por las directivas } \\
\text { y de conocimiento general de las áreas. }\end{array}$ \\
\hline
\end{tabular}

Fuente: elaborado por los autores.

\section{Anotaciones finales}

Es importante llamar la atención de que el proceso de convergencia a las NIIF no solamente consiste en cumplir con la ley y cambiar unas normas locales por unas internacionales, sino que para una institución educativa se constituye en una oportunidad para mejorar el manejo de los recursos y por lo tanto obtener una mayor calidad en el servicio que presta.

El proceso de implementación de las NIIF debe llevarnos a la reflexión de la importancia de la comunicación de las organizaciones con sus usuarios. En el caso de las instituciones educativas, se sabe que la sociedad entera las tiene como referentes y por lo tanto requiere de ellas información fiable y transparente con respecto a sus actividades para la prestación del servicio público de la educación. Sin embargo, en Colombia, poco se sabe sobre la información financiera de las instituciones universitarias, pues durante años este tema ha sido manejado con cautela, como sucede con casi todas las organizaciones de nuestro país, para las cuales la contabilidad es tema privado.

Al hacer una revisión sobre las universidades con gran reconocimiento en el mundo, como Harvard, Stanford y MIT, se encuentra que ellas publican sus reportes anuales en sus páginas web 
desde hace varios años. En los países latinoamericanos, y especialmente en Colombia, eso es una utopía. Sin embargo, desde la academia contable se debe insistir en la importancia de tener en cuenta las necesidades de los usuarios externos, en especial cuando se trata de entidades que, por sus características implícitas, tienen un ámbito de usuarios amplio, como lo son las instituciones educativas y por lo tanto se requiere saber con gran detalle la forma en la cual desarrollan sus actividades para el bien de la sociedad y del servicio que prestan.

Con este trabajo de investigación se encontró que el proceso de implementación de NIIF en las instituciones educativas requiere una gran inversión en recursos económicos, humanos y tecnológicos, y depende en gran medida de la decisión con la cual las directivas de la institución tomen la iniciativa de realizar todas las tareas pertinentes, teniendo en cuenta que el área financiera tiene un papel primordial en los cambios, pero que todas las áreas deben involucrarse para lograr unos mejores resultados.

Las entidades universitarias cumplen un papel fundamental en la sociedad y por lo tanto deberían reconocer que cuentan con grupos de interés que van desde estudiantes, docentes y administrativos hasta padres de familia, egresados y comunidad en general. Como se ha visto en nuestro país, los errores que se cometen en estas organizaciones pueden afectar no solo el presente, sino el futuro de muchas personas. Por eso es importante que dichas entidades emprendan las acciones enmarcadas en el paradigma de la accountability ('responsabilidad pública') y en esa medida se preocupen por brindar información financiera y no financiera, cuantitativa y cualitativa para "informar a todos aquellos que se encuentran condicionados, afectados o que intervienen en las actuaciones de las organizaciones" (Católico, 2012, p. 61).

Se denota que el inicio de la aplicación de las NIIF en el país ha generado una gran reflexión sobre lo que se estaba realizando con respecto a la normatividad contable local. Para las entidades universitarias esto ha sido una realidad. Por una parte, se logró la expedición del Plan Único de Cuentas para IES (Resolución 643 de 2015 de la Contaduría General de la Nación), un avance, si se tiene en cuenta que dichas entidades habían tenido por años que adaptar el Plan Único de Cuentas para Comerciantes para el registro de sus operaciones. Por otra, se expidió la Ley 1740 de 2014, mediante la cual se regula la inspección y vigilancia de la Educación Superior y se destaca el Artículo 6, mediante el cual el Ministerio de Educación Nacional tendrá la facultad de "solicitar, confirmar y analizar en la forma, detalle y términos que determine, la información que requiera sobre la situación jurídica, contable, económica, administrativa o de calidad de cualquier institución de Educación Superior".

Asimismo, Giraldo, Abad y Díaz (2007) agregan que la autonomía universitaria otorgada también por la Constitución Nacional ha sido interpretada por algunas de las instituciones universitarias

como una libertad sin responsabilidad, produciendo un aumento exagerado de programas de pregrado y especialización, la gran mayoría de dudosa calidad. Es evidente que el crecimiento desbordado de programas se ha llevado a cabo, en muchos casos, sin tener en cuenta las necesidades reales de la comunidad educativa, sin un proceso de planeación adecuado, sin contar con recursos para prestar el servicio con niveles mínimos de calidad y, lo que es peor, con un claro y casi único propósito de lucro. (Giraldo, Abad \& Díaz, 2007, p. 3) 


\section{Referencias}

1. Arias-Bello, M. L. \& Sánchez-Serna, A. d. (2011). Valuación de activos: una mirada desde las Normas Internacionales de Información Financiera, los estándares internacionales de valuación y el contexto actual colombiano. Cuadernos de Contabilidad, 12 (30), 95-126.

2. Castańo-Ríos, C. E., Correa-García, J. A. \& ZamarraLondoño, J. E. (2014). Efectos financieros en una cooperativa colombiana por la implementación de estándares internacionales de información financiera para las pyme en su balance de apertura. Cuadernos de Contabilidad, 15 (38), 427-458.

3. Cañibano-Calvo, L. (2004). Información financiera y gobierno de empresa. Revista Internacional Legis de Contabilidad y Auditoría, 19, 157-235.

4. Católico, D. F. (2012). Revelación y divulgación de la información financiera y no financiera de las universidades públicas en Colombia. Revista de Ciencias Económicas, 20 (1), 57-76.

5. Consejo Técnico de la Contaduría Pública. (26 de agosto de 2008). Concepto 015 [documento en línea]. Recuperado de http://actualicese.com/normatividad/2008/08/26/concepto-015-de-26-08-2008/

6. Consejo Técnico de la Contaduría Pública. (2015a). Documento de Orientación Técnica No. 5 - Orientaciones Técnicas sobre la Aplicación de las NIIF para Pymes [Serie de Información Financiera y Contabilidad - Convergencia con las Normas Internacionales de Información Financiera (NIIF) en Colombia].

7. Consejo Técnico de la Contaduría Pública. (2015b). Orientación Técnica N. ${ }^{\circ}$ 14. Entidades Sin Ánimo de Lucro. Documento de Orientación Técnica. Bogotá, Colombia.

8. Cuevas-Mejía, J. J. \& Usme-Suárez, W. A. (2013). Aproximación al rol de la información financiera elaborada con fines externos en un contexto de hibridaje cultural. Cuadernos de Contabilidad, 14 (36), 1045-1078.

9. Díaz Henao, J. U. (2014). Convergencia a las NIIF en Colombia. Reglamentación y perspectivas. Apuntes contables, 17, 271-283.
10. Ferrer de la Hoz, M. A. (2013). Análisis del proceso de convergencia a Normas Internacionales de Contabilidad e Información Financiera desde los factores intrínsecos al sistema contable en Colombia. Cuadernos de Contabilidad, 14 (36), 971-1007.

11. Giraldo G., U., Abad A., D. \& Díaz P., E. (2007). Bases para una politica de calidad en la Educación Superior en Colombia. CNA.

12. Hernández Carrera, R. (2014). La investigación cualitativa a través de entrevistas: Su análisis mediante la teoría fundamentada. Cuestiones Pedagógicas, 23, 187-210.

13. Mantilla Blanco, S. A. (2014). IFRS / NIIF Entendiendo lo básico. Bogotá: SAMantilla.

14. Martín Vallespin, E. (2009). Rendición de cuentas en la educación superior: Un análisis de las cuentas anuales de las universidades en España y el Reino Unido. Contaduría, 55, 75-101.

15. Misas Arango, G. (2004). La Educación Superior en Colombia - Análisis y estrategias para su desarrollo. Bogotá: Universidad Nacional de Colombia.

16. Pinto Prieto, L. P., Becerra Ardila, L. E. \& Gómez, L. C. (2013). Análisis del sistema de Gestión Financiera Sostenible de Instituciones de Educación Superior Públicas Colombiana. Revista de Educación en Ingeniería, 8 (15), 11.

17. Ramírez Pinzón, J. F. (2015). Análisis de los efectos no financieros de la Implementación de las NIIF: mirada a algunas experiencias internacionales. Una mini revisión de literatura (tesis de pregrado). Pontifica Universidad Javeriana, Facultad de Ciencias Económicas y Administrativas.

18. Roa Varelo, A. \& Pacheco, I. (Eds.). (2014). Educación Superior en Colombia - Doce propuestas para la próxima década. Barranquilla: Editorial Universidad del Norte.

19. Salazar Baquero, E. E. (2011). Análisis de las implicaciones no financieras de la aplicación de la NIIF para pyme en las medianas entidades en Colombia. Cuadernos de Contabilidad, 12 (30), 211-241. 